\title{
Maternal influence on the fetal microbiome in a population-based study of the first-pass meconium
}

\author{
Terhi Tapiainen ${ }^{1}$, Niko Paalanne ${ }^{2}$, Mysore V Tejesvi ${ }^{3}$, Pirjo Koivusaari ${ }^{3}$, Katja Korpela ${ }^{2}$, Tytti Pokka ${ }^{2}$, Jarmo Salo ${ }^{2}$, \\ Tuula Kaukola ${ }^{2}$, Anna Maria Pirttilä ${ }^{3}$, Matti Uhari ${ }^{1}$ and Marjo Renko ${ }^{1}$
}

BACKGROUND: Meconium is formed before birth and may reflect the microbiome of the fetus. To test our hypothesis, we investigated whether maternal factors during pregnancy, such as biodiversity of the living environment, influence the microbiome of the first stool more than immediate perinatal factors.

METHODS: We recruited 218 consecutive newborn infants from one hospital. Regions of the bacterial 16S rRNA gene were sequenced to characterize the microbiomes of the firstpass meconium samples $(N=212)$. We used a multivariate model to determine both the prenatal and perinatal factors affecting the microbiome.

RESULTS: The number of operational taxonomic units ranged from 0 to 448 per newborn. The most abundant phyla were Firmicutes, with a relative abundance of $44 \%$, Proteobacteria, $28 \%$, and Bacteroidetes, $15 \%$. By a multivariate analysis, the biodiversity of the home environment increased the diversity of microbiomes, whereas perinatal factors, such as the delivery mode or exposure to antimicrobials during labor did not have an effect.

CONCLUSION: The microbiome of the first-pass meconium was not altered by immediate perinatal factors, but was affected by maternal factors during pregnancy, implying the in utero transfer of microbes and the development of the gut microbiota niche in fetal life.

$T$ he first steps of bacterial colonization after birth induce long-term effects on the immunological system of the child, as shown in studies comparing infants born vaginally to those born through cesarean section (C-section). Before birth, however, the human fetus may frequently encounter nonpathogenic bacteria. Culture-independent methods, such as the next-generation sequencing of bacterial $16 \mathrm{~S}$ small-subunit ribosomal gene (16S rRNA), have detected bacterial DNA of several bacterial species, both in amniotic fluid and in the umbilical cord (1). The magnitude and the biological effects of such intrauterine bacterial exposure before birth are largely unknown. Yet, for instance, bacterial DNA found in culture negative amniotic fluid has been associated with placental inflammation known to increase the risk of preterm birth (2). The sampling of amniotic fluid, however, requires invasive methods and cannot be used to study prenatal bacterial exposure in normal pregnancies to elucidate the bacterial contacts of the fetus and their biological effects before birth.

As the first-pass meconium, i.e., the first stool after birth is formed before birth from the amniotic fluid, mucus, and epithelial cells, its microbiome could reflect the bacterial exposure of the fetus during pregnancy. Several studies during the last 10 years have shown that the first-pass meconium contains measurable microbial DNA of several bacterial species (3,4,5-11). Most studies, however, have focused mainly on the first stool samples of preterm infants, and have included only a small number of newborns ranging from 4 to 52 infants per study.

To test the hypothesis that the microbiome of the first-pass meconium could reflect the bacterial exposure of the fetus before birth, we designed a large population-based cohort study and examined the associations between the microbiome of the first-pass meconium and environmental exposures during pregnancy and delivery.

\section{METHODS}

\section{Study Design and Population}

For this population-based study, we recruited consecutive newborn infants born in Central Finland Central Hospital in Jyväskylä, Finland, between 3rd February 2014 and 13th March 2014. The hospital serves as the sole primary delivery hospital for a population of 250,000 people, with about 3,000 annual births. We invited all mothers delivering during that period to participate. Nurses in the maternity ward handed out the information letters to parents, and all the parents of the participants gave their written informed consent. The Ethics Committee of the Central Finland Hospital District, Finland, found the study plan ethically acceptable.

\section{Data and Stool Sample Collection at Birth}

The parents completed a maternal medical history questionnaire, including items on the consumption of probiotics and antimicrobials during pregnancy, and the nurses completed another questionnaire about pregnancy and birth (Table 1). The midwife collected the first spontaneously evacuated meconium from the diaper of each

\footnotetext{
'Department of Pediatrics, PEDEGO Research Unit and Medical Research Center Oulu, University of Oulu, Oulu, Finland; ${ }^{2}$ Department of Children and Adolescents, Oulu University Hospital, Oulu, Finland; ${ }^{3}$ Department of Genetics and Physiology, Faculty of Science, University of Oulu, Oulu, Finland. Correspondence: Terhi Tapiainen (terhi.tapiainen@oulu.fi)

Received 20 June 2017; accepted 29 January 2018; advance online publication 2 May 2018. doi:10.1038/pr.2018.29
} 
Table 1. Baseline characteristics of the population in whom the first stool obtained after birth was studied $(N=212)$

\begin{tabular}{lc}
\hline Characteristic & $N(\%)$ \\
\hline Gender & \\
Male & $114(54)$
\end{tabular}

\section{Mother's education}

Junior high school

Senior high school

Vocational school

Polytechnic

University

Number of older siblings

None

One

Two or more

Maternal use of probiotics ${ }^{a}$

Presence of furry pets at home

\section{Mode of delivery}

Vaginal

Cesarean section

Antibiotics during delivery ${ }^{\mathrm{b}}$

Gestational age, mean wk (range)

$40(35-42)$

Birth weight, mean $\mathrm{g}$ (range)

$3,553(2,220-4,960)$

Sample collection age, mean $\mathrm{h}$ (range)

$8.1(0-32)$

${ }^{a}$ Any use of commercially available probiotic products containing lactobacilli.

${ }^{b}$ Cefuroxime $(n=31)$, penicillin $(n=28)$, and piperacillin-tazobactam $(n=2)$.

newborn infant and put it into two sample tubes. Similar sample tubes and diapers were used throughout the study. All meconium samples were immediately cooled and kept at refrigerator temperature $\left(+4\right.$ to $\left.+8{ }^{\circ} \mathrm{C}\right)$ for not more than $24 \mathrm{~h}$. The samples were then frozen at less than $-22^{\circ} \mathrm{C}$ and transferred to the University of Oulu, Finland for microbiome analysis, performed by $16 \mathrm{~S}$ rRNA gene sequencing.

\section{DNA Extraction and Amplification of Bacterial rRNA Genes}

DNA was extracted from the meconium samples using the QIAsymphony DSP DNA Mini Kit, according to the manufacturer's protocol (Qiagen), and stored at $-20{ }^{\circ} \mathrm{C}$ until used. The DNA was quantified using a Nanodrop spectrophotometer. A portion of the $16 \mathrm{~S}$ small-subunit ribosomal gene was amplified using primers F519 (5'-CAGCMGCCGCGGTAATWC-3') and R926 (5'CCGTCAATTCCTTTRAGTTT- $3^{\prime}$ ). The F519 primer contained an Ion Torrent pyrosequencing adapter sequence A (Thermo Fisher Scientic, MA, USA), a 9-bp unique barcode sequence, and one nucleotide linker, whereas the R926 primer contained an Ion Torrent adapter trP1 sequence. PCR reactions were performed in three replicates, each containing $1 \times$ Phusion GC buffer, $0.4 \mu \mathrm{M}$ of the forward and reverse primers, $200 \mu \mathrm{M}$ dNTPs, $0.5 \mathrm{U}$ of Phusion enzyme (Finnzymes, Finland), and $10 \mathrm{ng}$ of genomic community DNA as the template, together with molecular-grade water in a total reaction volume of $25 \mu$ l. The cycling conditions were 35 cycles at $98^{\circ} \mathrm{C}$ for $10 \mathrm{~s}, 64{ }^{\circ} \mathrm{C}$ for $30 \mathrm{~s}$, and $72{ }^{\circ} \mathrm{C}$ for $20 \mathrm{~s}$ after an initial denaturation at $98^{\circ} \mathrm{C}$ for $3 \mathrm{~min}$. After the PCR amplification, the pooled triplicate reactions were purified using the AMPure XP PCR clean-up kit (Agencourt Bioscience, CA), and DNA concentration was measured on a Bioanalyzer DNA chip (Agilent Technologies, CA). For sequencing, each individual sample was pooled in an equivalent amount, the sample was size-selected on $1.5 \%$ agarose gel using the BluePippin automated electrophoresis system (Sage Science, MA), purified twice with the AMPure XP kit, and the final DNA concentration was measured on a Bioanalyzer DNA chip. The sequencing sample was diluted to $15 \mathrm{pM}$ and sequenced on a $316 \mathrm{v} 2$ chip, using an Ion Torrent $400 \mathrm{bp}$ chemistry (Thermo Fisher Scientic).

\section{Bioinformatics Analysis}

The hypervariable regions V4-V5 of the 16S rRNA gene were sequenced using an Ion Torrent to characterize the microbiomes of the meconium samples. The Ion Torrent sequences were processed and analyzed with QIIME, using state-of-the-art procedures (12). Briefly, the sequences were binned according to sample-specific barcodes by means of the QIIME split_libraries.py tool, after which the barcode and the primer sequences were trimmed and filtered for quality using the default parameters. Chimeric sequences were removed with the Usearch quality filtering tool in QIIME using the rRNA16S.gold.fasta reference database (13). The final data set consisted of 4.94 million readings from 197 samples after filtering out low-quality and chimeric readings, with a median of 24,423 readings per sample. As including samples containing fewer than 1,000 readings can result in false-positive findings (14), we removed samples containing fewer than 1,000 readings. The sequences were clustered into operational taxonomic units (OTUs), employing a similarity threshold of $97 \%$ in a RDP Naive Bayesian Classifier with a score filtering threshold of 0.5. The OTUs represent taxonomic units based on the differences between sequences of bacterial DNA data. The total number of OTUs serve as an estimate of the total number of bacterial species in the sample, but is not an exact equivalent of the number of different microbiological species. The phylogenetic trees were constructed from NAST-trimmed aligned sequences in FastTree2 (15). The rarefaction, relative abundance, and core microbiome analysis were all performed with QIIME. The detailed taxonomic composition of the bacteria from phylum to species level was visualized with Krona, Genus, and species information was retrieved from the OTU table using the "Summarize taxa" command in QIIME and taken as input data for Krona (16). We used principal co-ordinate analysis (PCoA), using QIIME, on the basis of calculations of the phylogenetic distances between the samples, using weighted UniFrac metrics PCoA, to describe the microbiomes with respect to the prenatal and perinatal factors. We have deposited the raw Ion Torrent data in NCBI-SRA with the accession numberSRP069890. The Shannon-Weaver index was used to estimate the diversity of the microbiome.

\section{Statistical Analysis}

To perform a quantitative statistical analysis of the raw microbiome data, we created sum variables for relative abundances of phyla and genera in SPSS software usingthe data received from the bioinformatics analysis. For instance, the relative abundances of all lactobacilli were combined in one variable, representing the genus Lactobacillus. Samples with fewer than 1,000 readings were coded as zero for all relative abundance values. We first described the general characteristics of the intestinal microbiome in a quantitative manner by calculating the mean relative abundance (\%) of the most abundant phyla and genera and standard deviations (SDs) for the relative abundances. We chose to use the mean relative abundance of the phylum Firmicutes and bacteria belonging to the genera Lactobacillus, Bifidobacterium, and Feacalibacterium, as these bacteria have earlier been associated with the health in infants and children (17-19). The genus Staphylococcus was included, as this was the most abundant genus in the samples. As we planned our statistical comparisons based on the previous findings of selected 
genera and phyla in the microbiome research, we chose to use $P$-level 0.05 as the level of statistical significance as we tested the preexisting hypotheses in our data set. We did not perform multiple comparisons outside the pre-existing hypotheses. In addition, we planned a multivariate analysis to adjust for possible confounders. The relative abundances of the pre-selected phyla and genera were compared with respect to the mode of delivery and the antimicrobial exposure. In addition, we compared the numbers of OTUs and the Shannon diversity indices with respect to the mode of delivery and the antimicrobial exposure. To compare the differences in background characteristics between the newborns with fewer than 1,000 readings in their meconium samples and those with more than 1,000 readings, we used the Pearson Chi-square test for all the binomial variables and a t-test for the gestational age. We used the MannWhitney $U$-test for the univariate analyses of relative abundances. We then used a multivariate general linear model to analyze factors affecting the microbiome. The mode of delivery, the use of antimicrobials during pregnancy or labor, the use of products containing lactobacilli during pregnancy, and the presence of furry pets at home during pregnancy were simultaneously included in the GLM model. The statistical analyses were performed with SPSS 22 software (SPSS, Chicago, IL).

\section{Quality Control Analysis}

Bacteria of the phylum Proteobacteria have recently been reported to be a major contaminant during DNA extraction in samples with low amounts of bacterial DNA $(20,21)$. In some samples, an untrustworthily high proportion of Rhodanobacter genus was noted in our study. Thus, for control purposes, the bacterial DNA was extracted in duplicate using both a QIAsymphony DSP DNA Mini Kit and a QIAamp Fast DNA Stool Mini Kit (Qiagen) from 15 samples. In addition, the bacterial DNA was extracted from two empty tubes (used for collecting meconium), two diapers, water, and the DNA extraction buffer and quantified using a Nanodrop spectrophotometer. We were not able to extract any DNA from the diapers or the stool sample kits. However, we were able to show that the DNA extraction kit and the QIAsymphony DSP DNA Mini Kit were

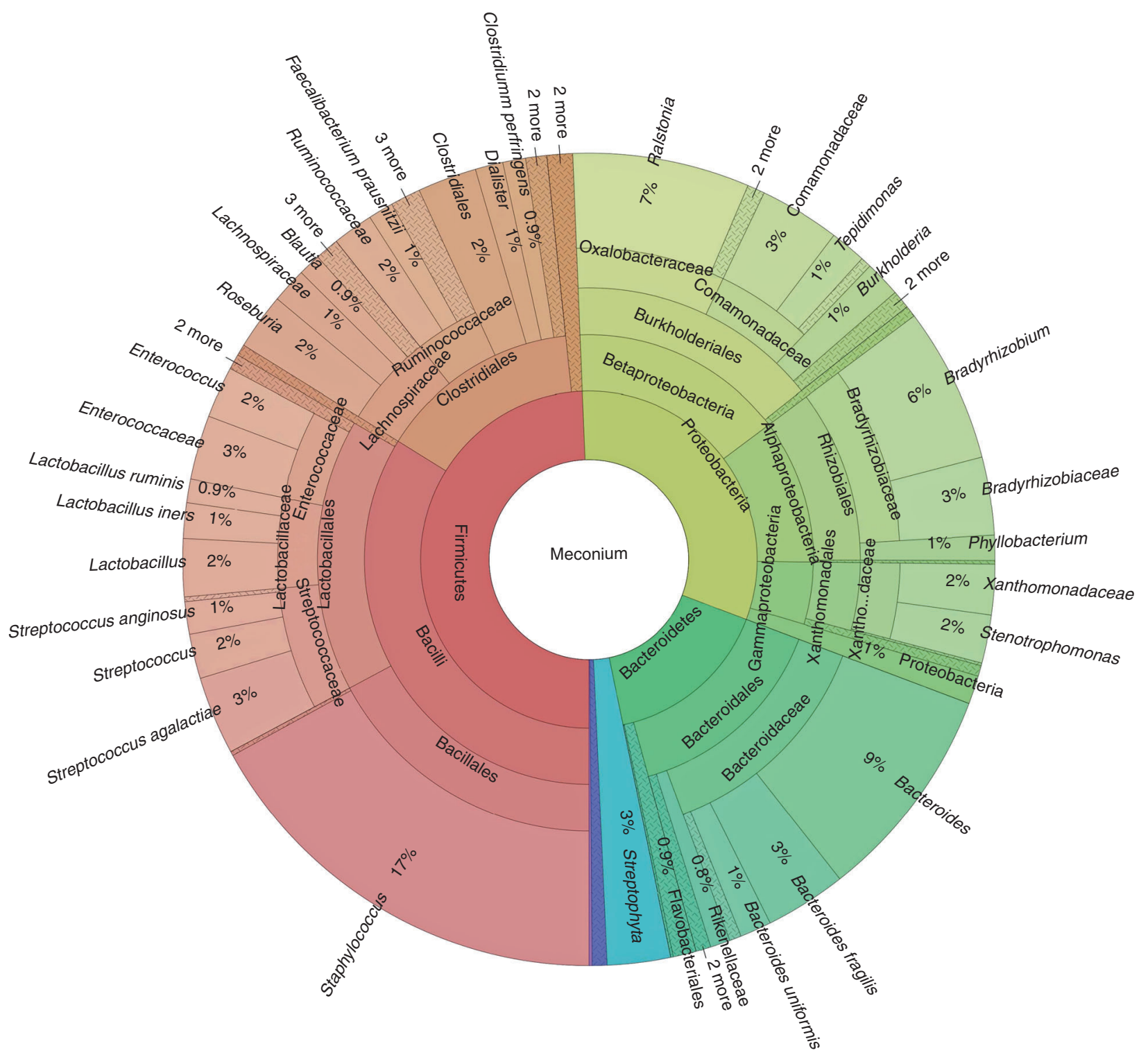

Figure 1. The relative abundance of gut microbiome in the first stool after birth in samples of 212 consecutive newborn infants born in the same hospital. Circles indicate different taxonomic ranks and columns indicate major distinct taxa with relative abundances $>1 \%$. Figure has been created using the Krona visualization package for metagenomics. 
contaminated by Rhodanobacter genus. Rhodanobacter does not belong to the human gut microbiome, but has been isolated from soil and groundwater. Thus, we removed Rhodanobacter from the data set in the bioinformatics analysis.

\section{RESULTS}

A total of 312 children were born during the defined period, and the families of 218 children (69\%) gave their informed consent. The first stool was sampled in 212 cases (97\%). The population consisted of near-term and term newborns with gestational ages ranging from 35 to 42 weeks (mean 39.6, SD 1.4) (Table 1). Four of them were born before gestational week 37. Altogether, 26 out of the 40 children born via C-section $(65 \%)$ and 33 of the 172 born vaginally (19\%) were exposed to

Table 2. The most abundant bacterial genera in the first stool of life in terms of the mean relative abundance of the genus in the wholebacterial DNA, as measured by the next-generation sequencing of bacterial 16SrRNA

\begin{tabular}{|c|c|c|c|}
\hline & Total $N$ & $\begin{array}{c}\text { Mean relative } \\
\text { abundance \% } \\
\text { (SD) }^{\mathrm{a}}\end{array}$ & $\begin{array}{c}\text { Proportion of } \\
\text { samples with the } \\
\text { specific genus } \\
N(\%)\end{array}$ \\
\hline \multicolumn{4}{|l|}{ Genera } \\
\hline Staphylococcus spp. & 212 & $14.9(26.5)$ & $136(64 \%)$ \\
\hline Bacteroides spp. & 212 & $12.0(19.3)$ & 75 (35\%) \\
\hline B. fragiles $^{\mathrm{b}}$ & 212 & $2.8(7.7)$ & $65(31 \%)$ \\
\hline B. uniformis ${ }^{\mathrm{b}}$ & 212 & $1.1(1.9)$ & $61(29 \%)$ \\
\hline Bradyrhizobium spp. & 212 & $8.1(12.8)$ & $130(61 \%)$ \\
\hline Ralstonia spp. & 212 & $6.2(11.7)$ & $130(61 \%)$ \\
\hline Streptococcus spp. & 212 & $5.4(11.7)$ & $132(62 \%)$ \\
\hline S. agalactiae ${ }^{\mathrm{b}}$ & 212 & $2.7(6.0)$ & 92 (43\%) \\
\hline S. anginosus ${ }^{\mathrm{b}}$ & 212 & $1.1(6.1)$ & $89(42 \%)$ \\
\hline Lactobacillus spp. & 212 & $4.1(13.7)$ & $129(61 \%)$ \\
\hline L. iners ${ }^{\mathrm{b}}$ & 212 & $1.2(8.5)$ & $31(15 \%)$ \\
\hline Enterococcus spp. & 212 & $4.0(17.4)$ & $81(38 \%)$ \\
\hline Comamonas spp. & 212 & $2.8(4.7)$ & $148(70 \%)$ \\
\hline Streptophyta spp. & 212 & $2.2(8.5)$ & $90(42 \%)$ \\
\hline Rosenburia spp. & 212 & $2.1(3.8)$ & $61(29 \%)$ \\
\hline Ruminicoccus spp. & 212 & $1.9(3.3)$ & $65(31 \%)$ \\
\hline Xantomonas spp. & 212 & $1.8(3.3)$ & $109(51 \%)$ \\
\hline Stenotrophomonas spp. & 212 & $1.7(4.2)$ & $115(54 \%)$ \\
\hline Clostridiales $^{c}$ & 212 & $1.6(2.7)$ & $65(31 \%)$ \\
\hline Clostridium spp. & 212 & $0.9(8.1)$ & $15(7 \%)$ \\
\hline Burkholderia spp. & 212 & $1.3(3.0)$ & $120(57 \%)$ \\
\hline Faecalibacterium spp. & 212 & $1.3(2.3)$ & $60(28 \%)$ \\
\hline Lachnospira spp. & 212 & $1.3(2.4)$ & $63(30 \%)$ \\
\hline Tepidimonas spp. & 212 & $1.3(3.1)$ & $99(47 \%)$ \\
\hline Dialister spp. & 212 & $1.0(1.8)$ & $59(28 \%)$ \\
\hline
\end{tabular}

All genera with a relative abundance of more than $1.0 \%$ are presented asamples with fewer than 1,000 readings were coded as zero.

${ }^{b}$ Species with a relative abundance of $1.0 \%$ or more are also presented. The abundances of these species are included in the relative abundances of the genera.

cOrder of unspecified Clostridiales, also including Clostridium spp. antimicrobials during delivery. The microbiomes of the first stool were diverse and showed high inter-individual variability (Figure 1). The most abundant phyla were Firmicutes, with a relative abundance of $44 \%$, Proteobacteria, 28\%, and Bacteroidetes, $15 \%$, whereas the most abundant bacterial genera were Staphylococcus, with a relative abundance of $15 \%$, followed by Bacteroides, 12\%, Streptococcus, $5.4 \%$, and Lactobacillus, $4.1 \%$, in the first stool. (Table 2).

In a total of 19 samples, DNA did not amplify sufficiently, i.e., there were fewer than 1,000 readings per sample. As the lack of amplification may imply that bacterial DNA was lacking, we first tested whether the samples of newborn infants with fewer than 1,000 readings were different, in terms of the prenatal and perinatal factors. Newborns with meconium samples with $<1,000$ readings $(N=19)$ and those with $>1,000$ readings $(N=193)$ did not differ statistically significantly with respect to gestational age, the mode of delivery, the use of antimicrobials during delivery, or the time of sampling after birth. However, maternal antimicrobial consumption during pregnancy significantly increased the proportion of meconium samples with $<1,000$ readings from 7 to $19 \%, P=0.03$.

The number of OTUs, using a similarity threshold of $97 \%$, ranged from zero to 448 per newborn. The number of OTUs observed in samples was not associated with the delivery mode or the time of sampling (Figure 2, Tables 3
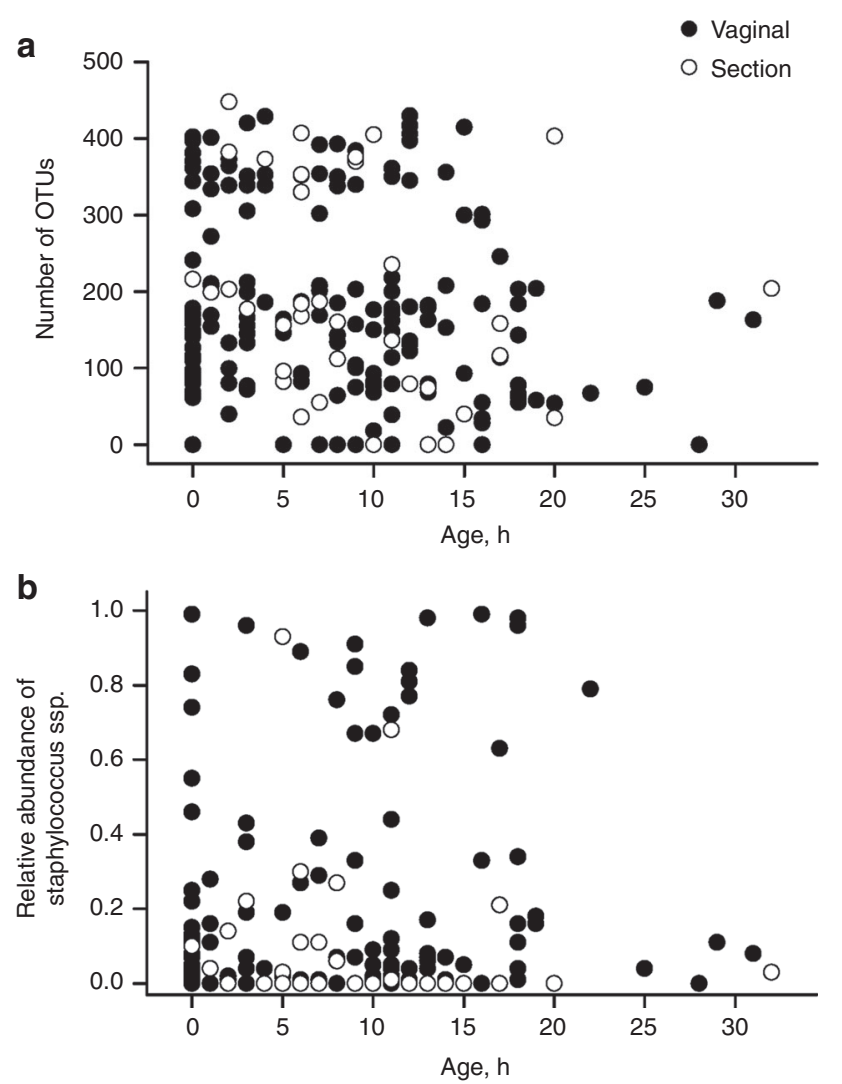

Figure 2. The effect of samping time on the microbiome in the firstpass meconium in infants born vaginally or via C-section. (a) The effect on the total number of operational taxonomic units (OTUs). (b) The effect on the relative abundance of Staphylococcus spp. 
Table 3. Effects of delivery mode, antimicrobial exposure at birth, maternal consumption of probiotics during pregnancy, and the presence of furry pets at home on the microbiome of the first stool

\begin{tabular}{|c|c|c|c|c|c|}
\hline Mode of delivery & Vaginal $(N=172)$ & & Section $(N=40)$ & & $P$ value \\
\hline Number of OTUs, mean (SD) ${ }^{a}$ & $177(122)$ & & 188 (139) & & 0.64 \\
\hline Firmicutes mean \% (SD) & $46(33)$ & & $36(30)$ & & 0.12 \\
\hline Staphylococcus spp. mean \% (SD) & $16(28)$ & & $9(19)$ & & $0.02^{*}$ \\
\hline Lactobacillus spp. mean \% (SD) & $4.6(15)$ & & $1.7(2.9)$ & & 0.80 \\
\hline Antimicrobials during delivery ${ }^{\mathrm{b}}$ & $\begin{array}{c}\text { No } \\
N=151\end{array}$ & & $\begin{array}{c}\text { Yes } \\
N=61\end{array}$ & & $P$ value \\
\hline Number of OTUs, mean (SD) ${ }^{a}$ & $178(124)$ & & $183(129)$ & & 0.62 \\
\hline Lactobacillus spp. mean \% (SD) & $3.3(12)$ & & $5.9(18)$ & & 0.40 \\
\hline Bifidobacterium spp. mean \% (SD) & $0.001(0.01)$ & & $0.007(0.03)$ & & $0.025^{*}$ \\
\hline Faecalibacterium spp. mean \% (SD) & $1.3(2.3)$ & & $1.3(2.3)$ & & 0.89 \\
\hline Maternal consumption of probiotics & $\begin{array}{c}\text { No } \\
N=151\end{array}$ & & $\begin{array}{c}\text { Yes } \\
N=61\end{array}$ & & $P$ value \\
\hline Number of OTUs, mean (SD) ${ }^{a}$ & $189(127)$ & & 155 (118) & & 0.11 \\
\hline Firmicutes mean \% (SD) & 151 & $47(32)$ & 61 & $37(33)$ & 0.027 \\
\hline Bacteroidetes mean \% (SD) & 151 & $16(22)$ & 61 & $12(19)$ & 0.069 \\
\hline Staphylococcus spp. mean \% (SD) & 151 & $16(28)$ & 61 & $12(23)$ & 0.50 \\
\hline Firmicutes mean \% (SD) & $48(35)$ & & $140(29)$ & & 0.14 \\
\hline Bacteroidetes mean \% (SD) & $11(18)$ & & $19(23)$ & & $0.006^{* *}$ \\
\hline Staphylococcus spp. mean \% (SD) & $19(31)$ & & $10(20)$ & & $0.046^{*}$ \\
\hline Lactobacillus spp. mean \% (SD) & $4.8(15)$ & & $3.3(12)$ & & 0.38 \\
\hline Bifidobacterium spp. mean \% (SD) & $0.002(0.02)$ & & $0.003(0.02)$ & & 0.65 \\
\hline Faecalibacterium spp. mean \% (SD) & $0.9(2.00)$ & & $1.7(2.6)$ & & $0.044^{*}$ \\
\hline \multicolumn{6}{|c|}{$\begin{array}{l}\text { The results of univariate analyses are presented. The number of operational taxonomic units (OTUs) may be taken as an estimate of the number of bacterial species present in } \\
\text { the sample, but is not microbiologically the same concept. Phylum level comparisons for mean relative abundances }(0-100 \%) \text { of Firmicutes and Bacteroidetes phyla and } \\
\text { genum level comparisons for relative abundances }(0-100 \%) \text { of Staphylococcus spp., Lactobacillus spp., Bifidobacterium spp., and Faecalibacterium spp. genera are presented. } \\
\text { aSamples fewer than } 1,000 \text { readings were coded as zero. } \\
\text { 'The groups overlap, i.e., } 26 \text { of the } 40 \text { children born via } C \text {-section }(65 \%) \text { and } 35 \text { of the } 172 \text { children born vaginally (20\%) were exposed to antimicrobials during delivery. The } \\
\text { antimicrobials that were used during delivery: cefuroxime }(n=31) \text {, penicillin }(n=28) \text {, piperacillin-tazobactam }(n=2) \text {. } \\
{ }^{*} \text { Mann-Whitney U-test; } P<0.05^{* *} \text { Mann-Whitney U-test } P<0.01 \text {. }\end{array}$} \\
\hline
\end{tabular}

and 4). The relative abundance of staphylococcal genera was not associated with the sampling time (Figure 2). As we observed no clear clusters in PCoA (Figure 3), we then continued for specific comparisons.
By univariate analysis, vaginal delivery increased, whereas the exposure to antibiotics during delivery decreased the relative abundance of Staphylococcus spp. (Table 3). The consumption of probiotic lactobacilli during pregnancy 


\section{Articles | Tapiainen et al.}

Table 4. Multivariate analysis of maternal factors and the microbiome of the first stool after birth is presented

\begin{tabular}{|c|c|c|c|c|}
\hline & Mode of delivery(vaginal birth) ${ }^{a}$ & Antimicrobials during labor & Probiotics (lactobacilli) during pregnancy & Furry pets at home \\
\hline \multicolumn{5}{|c|}{ Number of OTUs } \\
\hline$\beta(95 \% \mathrm{Cl})^{\mathrm{b}}$ & $-16(-63$ to 31$)$ & $1.7(-39$ to 42$)$ & $-33(-70$ to 4.6$)$ & 36 (2.0 to 69$)$ \\
\hline$P$ value & 0.50 & 0.93 & 0.086 & $0.038^{*}$ \\
\hline \multicolumn{5}{|c|}{ Bacterial diversity index ${ }^{c}$} \\
\hline$\beta(95 \% \mathrm{Cl})$ & $-0.28(-0.91$ to 0.34$)$ & $0.05(-0.48$ to 0.59$)$ & $-0.14(-0.64$ to 0.36$)$ & $0.53(0.09$ to 0.98$)$ \\
\hline$P$ value & 0.38 & 0.84 & 0.59 & $0.019^{*}$ \\
\hline \multicolumn{5}{|l|}{ Firmicutes } \\
\hline$\beta(95 \% \mathrm{Cl})$ & $0.84(-0.04$ to 0.21$)$ & $-0.04(-0.14$ to 0.07$)$ & $-0.10(-0.19$ to 0.001$)$ & $-0.09(-0.18$ to -0.004$)$ \\
\hline$P$ value & 0.17 & 0.50 & 0.053 & $0.040^{*}$ \\
\hline \multicolumn{5}{|l|}{ Bacteroidetes } \\
\hline$\beta(95 \% \mathrm{Cl})$ & $-0.04(-0.11$ to 0.03$)$ & $0.16(-0.05$ to 0.08$)$ & $-0.05(-0.11$ to 0.01$)$ & 0.07 (0.02 to 0.12$)$ \\
\hline$P$ value & 0.24 & 0.61 & 0.077 & $0.008^{* *}$ \\
\hline \multicolumn{5}{|c|}{ Staphylococcus spp. } \\
\hline$\beta(95 \% \mathrm{Cl})$ & $0.05(-0.05$ to 0.15$)$ & $-0.07(-0.15$ to 0.01$)$ & $-0.04(-0.12$ to 0.04$)$ & $-0.09(-0.16$ to -0.02$)$ \\
\hline$P$ value & 0.303 & 0.10 & 0.32 & $0.009^{*}$ \\
\hline \multicolumn{5}{|c|}{ Lactobacillus spp. } \\
\hline$\beta(95 \% \mathrm{Cl})$ & $0.05(-0.002$ to 0.10$)$ & $0.04(-0.003$ to 0.09$)$ & $0.006(-0.04$ to 0.05$)$ & $-0.02(-0.05$ to 0.02$)$ \\
\hline$P$ value & 0.060 & 0.065 & 0.77 & 0.37 \\
\hline \multicolumn{5}{|c|}{ Bifidobacterium spp. } \\
\hline$\beta(95 \% \mathrm{Cl})$ & $<0.001$ & $<0.0001$ & $<0.0001$ & $<0.0001$ \\
\hline$P$ value & 0.57 & 0.11 & 0.62 & 0.68 \\
\hline \multicolumn{5}{|c|}{ Faecalibacterium spp. } \\
\hline$\beta(95 \% \mathrm{Cl})$ & $-0.005(-0.01$ to 0.003$)$ & $-0.001(-0.01$ to 0.006$)$ & $-0.008(-0.02$ to -0.001$)$ & $0.007(0.001$ to 0.01$)$ \\
\hline$P$ value & 0.20 & 0.76 & $0.018^{*}$ & $0.02^{*}$ \\
\hline
\end{tabular}

OTU operational taxonomic unit, ${ }^{*} P<0.05,{ }^{*} P<0.01$.

The general linear model was adjusted for the mode of delivery, antimicrobials during labor, probiotics during pregnancy, and furry pets at home, i.e., all variables were included in the model at the same time. Positive $\beta$ coefficient indicates positive association, whereas negative $\beta$ coefficient indicates negative association. $P$ values present the sta-

tistical significance of the effect when all maternal variables were adjusted in the analysis.

${ }^{a}$ Cesarean section was used as a reference.

${ }^{b}$ Regression coefficient values $\beta<0$ indicate reduced and $\beta>0$ indicate increased relative abundance.

"Shannon diversity index.

slightly increased the abundance of the Lactobacillus genus in the first stool (Table 3).

By multivariate analysis, the perinatal factors, such as delivery mode and exposure to antibiotics during delivery were not associated with the observed properties of the microbiomes, such as the diversity of microbiomes, total number of OTUs, or relative abundances of main phyla or genera (Table 4). By multivariate analysis, the biodiversity of the prenatal maternal living environment, measured by the number of furry pets during the pregnancy, increased the diversity of microbiomes, the total number of OTUs, and the relative abundance of OTUs belonging to Bacteroidetes phylum and Fecalibacterium genus (Table 4) The maternal consumption of probiotics was not associated with the diversity of the microbiome in the first-pass meconium in multivariate analysis.

\section{DISCUSSION}

The microbiome of the first-pass meconium was not altered by immediate perinatal factors in our population-based cohort of newborn infants. Maternal antibiotics during pregnancy increased the number of samples that did not 

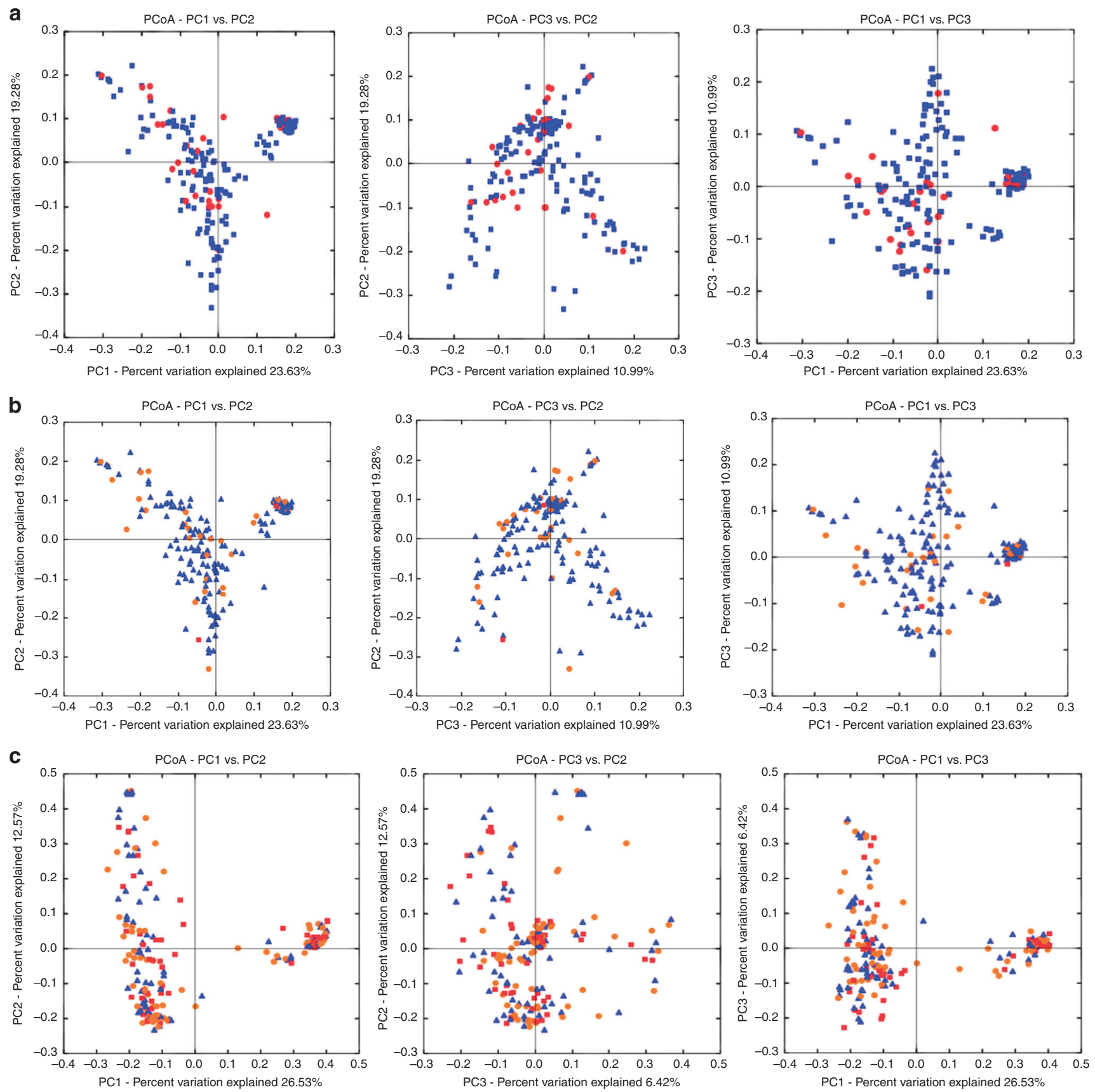

Figure 3. Principal co-ordinate analysis ( $P C O A)$ of perinatal and prenatal factors on the microbiome of the first-pass meconium. (a) Effects of delivery mode. Blue dots indicate vaginal birth and red dots Cesarean section. (b) Effect of maternal antibiotic exposure. Orange dots indicate infants exposed to maternal antibiotics and blue dots the unexposed. (c) Effect of biodiversity of the maternal living environment, i.e., the presence of furry pets in the family. Orange dots indicate the infants born to mothers with furry pets, blue dots the unexposed, and red dots infants with missing data in the survey, i.e. without active denial of furry pets.

amplify due to the possible lack of bacterial DNA in the firstpass meconium, whereas the greater biodiversity of maternal living environment during pregnancy increased the diversity of microbiome, implying the in utero transfer of microbes. Thus, the microbiome of the first stool after birth could be an indicator of the fetal microbiome and intrauterine bacterial exposure.
The maternal influence on fetal intestinal colonization observed in the present study is plausible in the light of earlier studies of bacterial transfer during pregnancy in animal models (22). In mice, the specifically labeled Enterococcus species given orally to pregnant mice was later detected in the intestinal meconium of pups obtained during C-section (23). Antibiotics during pregnancy have been shown to change the 


\section{Articles | Tapiainen et al.}

neonatal intestinal microbiome, resulting in poorer host resistance to Escherichia coli sepsis in neonatal mice (24). In humans, maternal mononuclear cells have been shown to carry whole bacteria or bacterial antigens more frequently during pregnancy, which have been suggested to be involved in imprinting the neonatal immune system (25). The microbiome of human placenta has recently been characterized and shown to include immunologically beneficial bacteria, such as Lactobacilli and Bifidobacteria (26,27). Although the mechanisms of in utero colonization of the fetus from the mother are poorly understood, it appears that the process is altered by maternal antibiotics, based on our present results. Whether these changes could have profounded immunological effects on the immune system of the newborns, as suggested in animal models (22), should be further investigated.

The greater biodiversity of the living environment has previously been associated with more diverse human microbiota (28). Children living in farms have been shown to have a decreased risk for allergies in adulthood (29). We had fewer than five children living in the farm in our study population, and were unable to analyze the effect of living in a farm on the first-pass meconium microbiome. Thus, we decided to use the exposure to furry pets during pregnancy, as a marker of the biodiversity of the maternal living environment. We observed that the presence of pets at home during pregnancy was associated with the biodiversity of the first-pass meconium in a multivariate model, i.e., regardless of the delivery mode, perinatal antibiotics, or consumption of probiotics. This is analogous to the previous studies, showing that exposure to furry pets was associated with the gut microbiome in neonates at the age of 1 and 4 months (30,31). According to our present study, the association between the environmental biodiversity and the human microbiome appears to exist before birth and is measurable in the first stool after birth.

Bacterial 16S rRNA gene sequencing is a fast and efficacious method that is suitable nowadays even for rather large sample sizes, as in this case, but it does not indicate the number of viable bacteria or the absolute number of bacteria detected. Yet, the presence of diverse microbiome in the first-pass meconium implies frequent contacts with bacterial DNA before birth, regardless of the viability of the bacteria. We performed sequencing of the hypervariable regions V4-V5 of the $16 \mathrm{~S}$ rRNA gene using IonTorrent platform, which is one of the leading sequencing platforms (32). The sequencing of several different $16 \mathrm{~S}$ rDNA regions have been used in $16 \mathrm{~S}$ community profiling in microbiome research during recent years (32). The error rate of IonTorrent platform against reference $16 \mathrm{~S}$ rRNA sequence is $1.5 \%$, which is in the range of error rates $1-2 \%$ reported using different platforms (32).

Most meconium samples reported before next-generation sequencing era previously have contained at least some cultivable bacteria, the most abundant genera being Staphylococcus, Streptococcus, Enterococcus, and Enterobacteriae, but the numbers of species detected in culture-based studies have been significantly lower than those obtained by the next-generation sequencing methods used here and in earlier studies $(7,23)$. Even though it would be logical to assume that the microbiome of the first stool, in life after birth by C-section, consisted of staphylococci and other skin bacteria; in the univariate analysis of our study, the Staphylococcus genus was the most abundant, particularly in infants born vaginally, possibly reflecting the first steps of postnatal skin and other colonization, that has reported to start earlier in vaginally delivered infants. The effects of delivery mode, however, disappeared in the multivariate analysis.

The strength of our study is the unselected, large, population-based sample of newborn infants with good clinical background data. Our sample size enabled us to perform multivariate analysis for environmental and maternal factors affecting the first steps of colonization and produce high-quality epidemiological data regarding maternal influence on fetal colonization. To ensure the quality of our microbiome analysis, we performed DNA extraction in duplicate in a subset of samples and confirmed that diapers or sampling tubes did not contain bacterial DNA. Thus, we were able to track the bacterial contamination present in one of the DNA isolation kits and control it in the bioinformatics analysis. We suggest that all microbiome studies on newborn colonization during the first days of life, when the amount of fecal bacterial DNA is still low, should be controlled using at least two different DNA extraction kits, to exclude the existing problem of bacterial DNA contamination leading to an excess of findings belonging to the Proteobacteria phylum in particular $(20,21)$. The limitation of our study is that the $16 \mathrm{~S}$ rRNA analysis is not a quantitative measurement, but analyzes the diversity of microbiomes. Thus, the differences shown in the present study are mostly based on the observed differences of relative abundances.

We conclude that the development of the gut microbiota niche starts in fetal life and is affected by maternal factors during pregnancy. Fetal exposure to bacteria during pregnancy can be estimated in a non-invasive manner, using the first-pass meconium samples as a proxy. The immunological effects of fetal microbiome should be further evaluated in the future.

\section{STATEMENT OF FINANCIAL SUPPORT}

The study was financially supported by the Juho Vainio Foundation, Finland, the Finnish Pediatric Research Foundation, and the Alma and K.A. Snellman Foundation, Finland.

Disclosure: The authors declare no conflict of interest.

\section{REFERENCES}

1. Jimenez E, Fernandez L, Marin ML, et al. Isolation of commensal bacteria from umbilical cord blood of healthy neonates born by cesarean section. Curr Microbiol 2005;51:270-4.

2. Han YW, Shen T, Chung P, Buhimschi IA, Buhimschi CS. Uncultivated bacteria as etiologic agents of intra-amniotic inflammation leading to preterm birth. J Clin Microbiol 2009;47:38-47.

3. Rouge $\mathrm{C}$, Goldenberg $\mathrm{O}$, Ferraris L, et al. Investigation of the intestinal microbiota in preterm infants using different methods. Anaerobe 2010;16: $362-70$. 
4. Mshvildadze M, Neu J, Shuster J, Theriaque D, Li N, Mai V. Intestinal microbial ecology in premature infants assessed with non-culture-based techniques. J Pediatr 2010;156:20-5.

5. Gosalbes MJ, Llop S, Valles Y, Moya A, Ballester F, Francino MP. Meconium microbiota types dominated by lactic acid or enteric bacteria are differentially associated with maternal eczema and respiratory problems in infants. Clin Exp Allergy 2013;43:198-211.

6. Stewart CJ, Marrs EC, Magorrian S, et al. The preterm gut microbiota: changes associated with necrotizing enterocolitis and infection. Acta Paediatr 2012;101:1121-7.

7. Moles L, Gomez M, Heilig H, et al. Bacterial diversity in meconium of preterm neonates and evolution of their fecal microbiota during the first month of life. PLoS ONE 2013;8:e66986.

8. Ardissone AN, de la Cruz DM, Davis-Richardson AG, et al. Meconium microbiome analysis identifies bacteria correlated with premature birth. PLoS ONE 2014;9:e90784.

9. Jakobsson HE, Abrahamsson TR, Jenmalm MC, et al. Decreased gut microbiota diversity, delayed Bacteroidetes colonisation and reduced Th1 responses in infants delivered by caesarean section. Gut 2014;63: 559-66.

10. Arboleya S, Sanchez B, Milani C, et al. Intestinal microbiota development in preterm neonates and effect of perinatal antibiotics. J Pediatr 2015;166: 538-44.

11. Hansen R, Scott KP, Khan S, et al. First-pass meconium samples from healthy term vaginally-delivered neonates: an analysis of the microbiota. PLoS ONE 2015;10:e133320.

12. Caporaso JG, Lauber CL, Walters WA, et al. Global patterns of $16 \mathrm{~S}$ rRNA diversity at a depth of millions of sequences per sample. Proc Natl Acad Sci USA 2011;108 (Suppl 1): 4516-22.

13. Edgar RC, Haas BJ, Clemente JC, Quince C, Knight R. UCHIME improves sensitivity and speed of chimera detection. Bioinformatics 2011;27:2194-200.

14. Ding T, Schloss PD. Dynamics and associations of microbial community types across the human body. Nature 2014;509:357-60.

15. Price MN, Dehal PS, Arkin AP. FastTree 2-approximately maximumlikelihood trees for large alignments. PLoS ONE 2010;5:e9490.

16. Ondov BD, Bergman NH, Phillippy AM. Interactive metagenomic visualization in a Web browser. BMC Bioinformatics 2011;12:385.

17. Savino F, Bailo E, Oggero R, et al. Bacterial counts of intestinal Lactobacillus species in infants with colic. Pediatr Allergy Immunol 2005;16:72-5.

18. Sokol H, Seksik P, Furet JP, et al. Low counts of Faecalibacterium prausnitzii in colitis microbiota. Inflamm Bowel Dis 2009;15:1183-9.
19. Pärtty A, Kalliomaki M, Endo A, Salminen S, Isolauri E. Compositional development of Bifidobacterium and Lactobacillus microbiota is linked with crying and fussing in early infancy. PLoS ONE 2012;7:e32495.

20. Grahn N, Olofsson M, Ellnebo-Svedlund K, Monstein HJ, Jonasson J. Identification of mixed bacterial DNA contamination in broad-range PCR amplification of $16 \mathrm{~S}$ rDNA V1 and V3 variable regions by pyrosequencing of cloned amplicons. FEMS Microbiol Lett 2003;219: $87-91$.

21. Salter SJ, Cox MJ, Turek EM, et al. Reagent and laboratory contamination can critically impact sequence-based microbiome analyses. BMC Biol 2014;12:87.

22. Romano-Keeler J, Weitkamp JH. Maternal influences on fetal microbial colonization and immune development. Pediatr Res 2015;77:189-95.

23. Jimenez E, Marin ML, Martin R, et al. Is meconium from healthy newborns actually sterile? Res Microbiol 2008;159:187-93.

24. Deshmukh HS, Liu Y, Menkiti OR, et al. The microbiota regulates neutrophil homeostasis and host resistance to Escherichia coli K1 sepsis in neonatal mice. Nat Med 2014;20:524-30.

25. Perez PF, Dore J, Leclerc M, et al. Bacterial imprinting of the neonatal immune system: lessons from maternal cells? Pediatrics 2007;119: e724-32.

26. Aagaard K, Ma J, Antony KM, Ganu R, Petrosino J, Versalovic J. The placenta harbors a unique microbiome. Sci Transl Med 2014;6:237ra65.

27. Satokari R, Grönroos T, Laitinen K, Salminen S, Isolauri E. Bifidobacterium and Lactobacillus DNA in the human placenta. Lett Appl Microbiol 2009;48:8-12.

28. Hanski I, von HL, Fyhrquist N, et al. Environmental biodiversity, human microbiota, and allergy are interrelated. Proc Natl Acad Sci USA 2012;109:8334-9.

29. Leynaert B, Neukirch C, Jarvis D, Chinn S, Burney P, Neukirch F. Does living on a farm during childhood protect against asthma, allergic rhinitis, and atopy in adulthood? Am J Respir Crit Care Med 2001;164 (10 Pt 1): 1829-34.

30. Nermes M, Endo A, Aarnio J, Salminen S, Isolauri E. Furry pets modulate gut microbiota composition in infants at risk for allergic disease. J Allergy Clin Immunol 2015;136:1688-90.

31. Azad MB, Konya T, Maughan H, et al. Infant gut microbiota and the hygiene hypothesis of allergic disease: impact of household pets and siblings on microbiota composition and diversity. Allergy Asthma Clin Immunol 2013;9:15.

32. D'Amore R, Ijaz UZ, Schirmer M. A comprehensive benchmarking study of protocols and sequencing platforms for $16 \mathrm{~S}$ rRNA community profiling. BMC Genomics 2016;17:55. 